\title{
Presentation / Presentación
}

The Network of Science and Technology for the Conservation of Cultural Heritage promotes the collaboration between the agents and stakeholders of the science-technology-business system, sharing experiences, knowledge, and technology with the main goal of contributing to the conservation and safeguard of Cultural Heritage.

The fourth edition of the International Congress on Science and Technology for the Conservation of Cultural Heritage was held in Seville, Spain, 26-30 March 2019, was focused on the application of digital and new technologies for the sustainable management, knowledge and social innovation for the prevention, conservation and management of heritage. More than 150 abstracts were received. The Congress had the support of Spanish National Research Council, Andalusian Institute of Historical Heritage, University Pablo de Olavide and University of Seville. And partially funded by the Spanish research projects

-ART-RISK: Artificial intelligence applied to preventive conservation of heritage buildings - a RETOS project of Ministerio de Economía y Competitividad and Fondo Europeo de Desarrollo Regional (FEDER), (code: BIA2015-64878-R; MINECO/FEDER-UE)

-TUTSOSMOD: Sustainable management of cultural heritage through BIM and GIS models: contribution to knowledge and social innovation. A project funded by the Ministry of Science, Innovation and Universities (HAR2016-78113-R)

This special issue includes papers from Technoheritage participants in the congress, based on the use of digital and new technologies, physico-chemical techniques or vulnerability assessment, among others. In this context, Science and Technology for the Conservation of Cultural Heritage have a key-role in research, establishing new methodologies and protocols towards better knowing of our historical buildings, archeological sites and artworks.
La Red de Ciencia y Tecnología para la Conservación del Patrimonio Cultural promueve la colaboración entre los agentes y las partes interesadas del sistema cienciatecnología-empresa, compartiendo experiencias, conocimientos y tecnología con el objetivo principal de contribuir a la conservación y salvaguarda del Patrimonio Cultural.

La cuarta edición del Congreso Internacional de Ciencia y Tecnología para la Conservación del Patrimonio Cultural, celebrada en Sevilla, España, del 26 al 30 de marzo de 2019, se centró en la aplicación de nuevas tecnologías digitales para la gestión sostenible, el conocimiento y la innovación social para la prevención, conservación y gestión del patrimonio. Se recibieron más de 150 resúmenes. El Congreso contó con el apoyo del Consejo Superior de Investigaciones Científicas de España, el Instituto Andaluz de Patrimonio Histórico, la Universidad Pablo de Olavide y la Universidad de Sevilla. Y estuvo parcialmente financiado por los proyectos de investigación españoles:

-RIESGO DE ARTE: Inteligencia artificial aplicada a la conservación preventiva de edificios patrimoniales: un proyecto RETOS del Ministerio de Economía y Competitividad y Fondo Europeo de Desarrollo Regional (FEDER), (código: BIA2015-64878-R; MINECO / FEDER-UE)

-TUTSOSMOD: Gestión sostenible del patrimonio cultural a través de modelos BIM y SIG: contribución al conocimiento y la innovación social. Un proyecto financiado por el Ministerio de Ciencia, Innovación y Universidades (HAR2016-78113-R)

Este número especial incluye artículos de los participantes del congreso Technoheritage, sobre el uso de las nuevas tecnologías digitales, técnicas fisicoquímicas o la evaluación de la vulnerabilidad, entre otros. En este contexto, la Ciencia y la Tecnología para la Conservación del Patrimonio Cultural tienen un papel clave en la investigación, estableciendo nuevas metodologías y protocolos para conocer mejor nuestros edificios históricos, sitios arqueológicos y obras de arte. 
During the Technoheritage conference, each presentation (oral or poster) was evaluated by two reviewers, and some authors were asked for publication in a special issue of Ge-Conservación. The final papers according the standard for authors of the journal, were also evaluated by double blind per review system.

We sincerely acknowledge the work of Technoheritage network, authors, Local Organizing Committee, Scientific Organizing Committee and the anonymous reviewers.

*Although all care is taken to ensure integrity and the quality of this publication and the information herein, no responsibility is assumed by the publishers, editors nor the authors for any damage to the property or persons as a result of operation or use of this publication and/or the information contained herein.

*The Editors declare that they have no conflicts of interest regarding the publication of this Special Issue.
Durante el congreso Technoheritage, cada presentación (oral o póster) fue evaluada por dos revisores, y se informó a algunos autores que la posibilidad de publicar en un número especial de Ge-Conservación. Los trabajos finales, según el estándar para autores de la revista, también fueron evaluados por un sistema doble ciego de revisión.

Quisiéramos agradecer sinceramente el trabajo de la red Technoheritage, los autores, el Comité Organizador Local, el Comité Organizador Científico y los revisores anónimos.

* Si bien se toman todas las precauciones para garantizar la integridad y la calidad de esta publicación y la información aquí contenida, los editores, editorial y autores no asumen ninguna responsabilidad por ningún daño a la propiedad o personas como resultado del funcionamiento o uso de esta publicación y/o la información aquí contenida.

* Los editores declaran que no tienen conflictos de intereses con respecto a la publicación de este número especial.

\section{Editores invitados}

\section{Javier Becerra Luna}

Universidad Pablo de Olavide, Sevilla, España

\section{Pilar Ortiz Calderón}

Universidad Pablo de Olavide,

Sevilla, España

\section{Francisco S. Pinto}

Universidad de Sevilla, Sevilla,

España

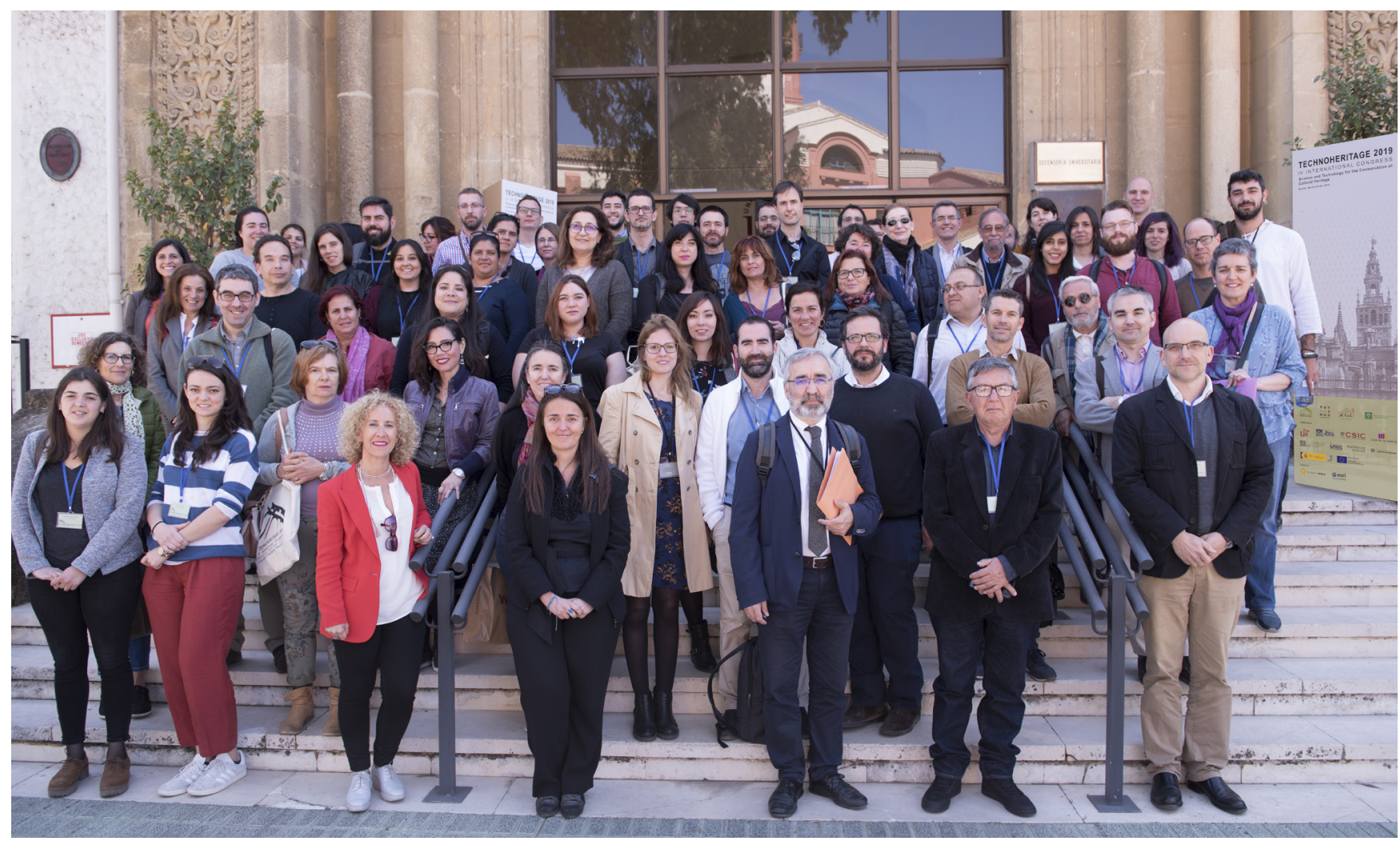

